\title{
The World Health Organization Disability Assessment Schedule-2.0 (WHODAS 2.0) in a chronic pain population being considered for chronic opioid therapy
}

This article was published in the following Dove Press journal:

Journal of Pain Research

\author{
Kelly M Wawrzyniak ${ }^{1,2}$ \\ Matthew Finkelman ${ }^{3}$ \\ Michael E Schatman 1,4 \\ Ronald J Kulich ${ }^{1,2}$ \\ Valerie F Weed ${ }^{5}$ \\ Eura Myrta' \\ David J DiBenedetto ${ }^{1,2}$
}

'Boston PainCare Center, Waltham, MA 0245I, USA; ${ }^{2}$ Department of Diagnostic Sciences, Tufts University School of Dental Medicine, Boston, MA 02III, USA; ${ }^{3}$ Division of Biostatistics and Experimental Design, Tufts University School of Dental Medicine, Boston, MA 02III, USA; ${ }^{4}$ Department of Public Health and Community Medicine, Tufts University School of Medicine, Boston, MA 02III, USA; ${ }^{5}$ Primary Care Psychology Associates, LLC Northbrook, IL 60062, USA
Correspondence: Kelly M Wawrzyniak Boston PainCare Center, 85 First Ave, Waltham, MA 0245I, USA

Tel +78 I 8957975

Fax +7812900893

Email kwawrzyniak@bostonpaincare.com
Purpose: To examine the validity of the World Health Organization Disability Assessment Schedule 2.0 (WHODAS 2.0) for the assessment of function in a community-based sample of patients with chronic pain conditions undergoing evaluation for chronic opioid therapy. Patients and methods: One hundred nine of 124 patients were evaluated for a chronic opioid therapy program between December 1, 2014 and April 10, 2015, inclusive, at one communitybased interdisciplinary pain management practice. Measures included: demographic data; the WHODAS 2.0; a modified version of the Roland Morris Disability Questionnaire (RMDQ-m); the Patient Health Questionnaire-9 item (PHQ-9); the Screener and Opioid Assessment for Patients with Pain-Revised (SOAPP-R); the Current Opioid Misuse Measure (COMM), the Opioid Risk Tool (ORT); opioid dose. These data were collected as part of routine care, and this retrospective chart review study examined the data from this convenience sample, comparing the results of each assessment tool to the results of the WHODAS 2.0.

Results: Median score on the WHODAS 2.0 was 25.69 (IQR=16.01 to 35.28). WHODAS 2.0 score was significantly correlated with the RMDQ-m $\left(\mathrm{r}_{\mathrm{s}}=0.69, p<0.001\right)$, the PHQ-9 $\left(\mathrm{r}_{\mathrm{s}}=0.68, p<0.001\right)$, the COMM $\left(\mathrm{r}_{\mathrm{s}}=0.52, p<0.001\right)$ and the SOAPP-R $\left(\mathrm{r}_{\mathrm{s}}=0.51, p<0.001\right)$. There was no significant correlation between the WHODAS 2.0 and the ORT $\left(\mathrm{r}_{\mathrm{s}}=0.14\right.$, $p=0.12)$ or opioid dose $\left(\mathrm{r}_{\mathrm{s}}=0.07, p=0.47\right)$.

Conclusions: The WHODAS 2.0 was significantly positively correlated with other measures, including measures of disability, risk of opioid misuse, and depression among patients being evaluated for chronic opioid therapy. The WHODAS 2.0 may be a useful measure of disability across a number of important domains when discussing expectations of both patients and providers at initiation of opioid therapy for chronic pain management. This assessment and discussion is crucial, particularly given the focus on function, rather than analgesia alone, when evaluating the effectiveness of opioid treatment.

Keywords: disability, chronic pain, opioids, function

\section{Introduction}

Chronic pain affects as many as 100 million Americans, ${ }^{1}$ resulting in an estimated cost to society of US\$635 billion annually, with costs due to lost productivity and related disability found to be as high as $\$ 335$ billion annually. ${ }^{2}$ With an estimated 13 million pain patients receiving long-term opioid treatment, ${ }^{3}$ chronic opioid therapy (COT) may contribute significantly to these costs. COT is reported to be associated with the development or exacerbation of substance use disorders, 
overdose/death, endocrinopathy, hyperalgesia, constipation, falls and fractures, motor vehicle accidents, cardiovascular events, and sleep apnea. ${ }^{4,5}$ The impact of COT on physical functioning is mixed, with some studies suggesting improvement and others describing diminishment across a range of domains. ${ }^{6-8}$ Accordingly, published guidelines have been increasingly calling for a shift of focus from pain reduction to functional improvement as the primary goal of COT. ${ }^{9}$ Unfortunately, assessing function in patients receiving COT is challenging as standardized, efficient, face valid measures that can be used across various pain conditions are generally lacking. Numerous self-report measures of disability and function with strong psychometric properties have been developed and/or validated in disease- and pain site-specific populations, including low back pain, ${ }^{10}$ neck pain, ${ }^{11}$ foot pain, ${ }^{12}$ osteoarthritis, ${ }^{13}$ fibromyalgia, ${ }^{14}$ rheumatoid arthritis, ${ }^{15}$ and migraine. ${ }^{16}$ Nonetheless, few disability measures have been validated across a variety of pain sites and conditions, with even fewer used cross culturally. Global measures such as the PROMIS PF CAT have received increasing attention in pain medicine; ${ }^{17}$ however, this group of measures has been challenged with respect to clinical utility. ${ }^{18}$

The World Health Organization Disability Assessment Schedule-2.0 (WHODAS 2.0) is a relatively short, facevalid measure of self-reported disability developed to measure disease burden across all psychiatric and medical diseases, and in all populations and cultures, ${ }^{19}$ and has been validated in populations suffering from myriad psychological and physical disease states. ${ }^{20}$ The WHODAS 2.0 assesses six domains of function that correspond to the "activity and participation" dimension of the International Classification of Functioning, Disability, and Health (ICF): Cognition (understanding \& communicating); mobility (moving \& getting around); self-care (hygiene, dressing, eating \& staying alone); getting along (interacting with other people); life activities (domestic responsibilities, leisure, work \& school); participation (joining in community activities). Administration of the 36-item version requires approximately 15-20 mins. This measure can be scored using simple scoring (a more practical way to hand-score for daily clinical use), or complex scoring based in item response theory. ${ }^{19}$

Despite the number of studies employing the WHODAS 2.0 , it has yet to be validated in a chronic pain patient population being considered for COT. As such, we present data examining the validity of the WHODAS 2.0 for the assessment of function in a community-based sample of patients with chronic pain conditions undergoing evaluation for COT.

\section{Material and methods}

This study was reviewed and determined by the Tufts University Health Sciences Institutional Review Board to be not human subject research due to the retrospective, deidentified data gathered per the protocol. As such, no informed consent process was done. Electronic health record data was extracted for a convenience sample of 124 subjects who were evaluated for a COT program between December 1, 2014 and April 10, 2015, inclusive, at one communitybased interdisciplinary pain management practice located in a suburb of Boston, Massachusetts. The evaluation process for COT program is standardized for all patients, and included a psychological evaluation, standardized self-report measures described below, self-reported functional goals, urine drug toxicology, and review of prescription drug monitoring program data. All of this information is gathered on one day, over the course of a $2.5 \mathrm{hr}$ evaluation with 3 specialist clinicians: psychologist/social worker, nurse practitioner, and chiropractor with expertise in functional assessment. This information is then presented in a weekly interdisciplinary team review meeting to determine risk of opioid misuse and potential benefit of COT, and finalize an interdisciplinary plan of care. Fifteen subjects' data were excluded from analyses due to apparent invalid or incomplete WHODAS 2.0 (Table 1), leaving 109 sets of data included in the data analysis.

\section{Variables}

Demographic data included age, gender, marital status, and work status (employed, retired, disabled, not employed, Worker's Compensation).

Table I Reasons for exclusion for 15 excluded subjects

\begin{tabular}{|l|l|}
\hline Reason & $\mathbf{n}$ \\
\hline Did not complete the evaluation visit & 2 \\
Family member completed questionnaires & 1 \\
No questionnaires completed due to blindness & 1 \\
Entire WHODAS 2.0 measure incomplete & 2 \\
Entire third page of WHODAS 2.0 measure incomplete & 3 \\
5 items of WHODAS 2.0 incomplete & 1 \\
4 items of WHODAS 2.0 incomplete & 3 \\
3 items of WHODAS 2.0 incomplete & 2 \\
\hline
\end{tabular}

Notes: ${ }^{a}$ incomplete item count does not include items in work/school section if left incomplete

Abbreviations: WHODAS 2.0, World Health Organization Disability Assessment Schedule 2.0. 


\section{Daily opioid dose}

Daily opioid dose at the time of evaluation was extracted from the electronic health record. The pain center's electronic health record includes customized clinical encounter templates for opioid prescribing evaluation visits, including a built in calculator to convert any opioid prescription entered into to daily milligrams morphine equivalent (MME) using the conversions published by the Washington State Agency Medical Directors' Group. ${ }^{21}$

\section{WHODAS 2.0}

The WHODAS 2.0 36-item version was used to assess disability. Each item uses a 5-point Likert scale to rate the degree of difficulty completing various tasks over the past 30 days: 1=None, 2=Mild, 3=Moderate, 4=Severe, $5=$ Extreme or Cannot Do. It has demonstrated high concurrent validity with comparable instruments designed to measure disability in day-to-day functioning across activity domains. ${ }^{19}$ Internal consistency is high for the total measure (Cronbach's $\alpha=0.96$ ) as well as each domain (Cronbach's $\alpha$ ranged from 0.79 to 0.98 ) and test-retest reliability was also good for the total measure (interclass correlation coefficient $=0.98$ ) as well as across items (interclass correlation coefficient ranged 0.69-0.89) and domains (interclass correlation coefficient ranged 0.930.96). ${ }^{19}$ The complex scoring method was used for this study wherein the summary score is converted to a metric ranging from $0=$ no disability to $100=$ full disability. The complex scoring program used for this study was downloaded from the WHO website (https://www.who.int/classi fications/icf/whodasii/en/), as advised in the WHODAS 2.0 manual. While the simple scoring method is recommended for daily clinical use, in part due to its ease with paper and pencil, the complex scoring method is recommended by WHO when conducting research.

\section{Additional self-report measures}

A modified version of the Roland Morris Disability Questionnaire (RMDQ-m) ${ }^{22}$ was used to assess disability due to pain. The 24-item measure was modified from each item describing "back pain" to "pain" (eg, "I walk more slowly than usual because of my back" was modified to "I walk more slowly than usual because of my pain"). Similar modifications to the items have been described in the literature and supported by the authors of the RMDQ. ${ }^{23}$ Scores range from 0 to 24 , with 0 representing no disability from pain and 24 representing maximum disability from pain.
The Patient Health Questionnaire-9 (PHQ-9) ${ }^{24}$ is a validated and reliable nine-item measure of the severity of depression symptoms. Scores range from 0 to 27 , with scores of 10 or greater indicating the need for further evaluation of a major depressive episode.

The Screener and Opioid Assessment for Patients with Pain-Revised (SOAPP-R) is a 24-item measure designed to predict opioid misuse in patients with chronic pain for whom initiation of COT is being considered. Each item is rated on a 5-point Likert scale anchored at 0 with "never" and at 4 with "very often". A score of 18 or greater is used to indicate higher risk of opioid misuse. Previous studies have shown good psychometric properties in comparable subject populations. ${ }^{25-27}$

The Current Opioid Misuse Measure (COMM) is a 17item measure assessing behaviors of patients on COT, with good reliability and validity reported in previous studies. ${ }^{28,29}$ A score of 9 or greater is used to indicate higher risk of opioid misuse.

The Opioid Risk Tool (ORT) $)^{30}$ is one of the measures completed by the behavioral provider using information gathered from the patient's self-report during the clinical interview. Scores range from 0 to 26 with a score of $0-3$ indicating low risk for opioid misuse, 4-7 as moderate risk, and 8 or greater as high risk.

\section{Statistical analyses}

Descriptive statistics (counts and percentages for categorical variables; medians, inter-quartile ranges, and ranges for discrete and continuous variables) were calculated. Associations between discrete/continuous variables were assessed via the Spearman rank-order correlation $\left(r_{s}\right)$ due to non-normality of data. Employment statuses were compared in terms of disability measures using the Kruskal-Wallis test; post-hoc testing was conducted via the Mann-Whitney U test with the Bonferroni correction. The significance level was set at $\alpha=0.05$, with the exception of tests in which the Bonferroni correction was employed. SPSS Version 24 was used in the analysis.

\section{Results}

Subjects included in data analysis $(\mathrm{n}=109)$ had a median age of 54 years (interquartile range $[\mathrm{IQR}]=48$ to 61 ) and ranged in age from 25 to 88 years. Forty-five percent were male and $71 \%$ were married or partnered. The median total score on the WHODAS 2.0 was 25.69 (IQR=16.01 to 35.28). Median scores and ranges for clinical variables and self-report measures are reported in Table 2. 
Table 2 Clinical characteristics for all subjects $(n=109)$

\begin{tabular}{|l|l|l|l|l|}
\hline Variable & Median & $\begin{array}{l}\text { First } \\
\text { Quartile }\end{array}$ & $\begin{array}{l}\text { Third } \\
\text { Quartile }\end{array}$ & Range \\
\hline MME & 15.0 & 0.0 & 62.0 & $0-400$ \\
PHQ-9 & 6.0 & 3.0 & 11.0 & $0-23$ \\
SOAPP-R & 14.0 & 9.0 & 21.0 & $0-70$ \\
COMM & 4.0 & 2.0 & 8.0 & $0-34$ \\
ORT & 2.0 & 1.0 & 5.0 & $0-16$ \\
RMDQ-m & 12.0 & 9.0 & 16.0 & $0-24$ \\
WHODAS 2.0 & 25.69 & 16.01 & 35.28 & $0-63.89$ \\
Communicating & 4.17 & 0.00 & 20.83 & $0-75.00$ \\
Getting & 50.00 & 30.00 & 65.00 & $0-90.00$ \\
Around & & & & \\
Self-Care & 12.50 & 0.00 & 25.00 & $0-75.00$ \\
Getting Along & 10.00 & 5.00 & 20.00 & $0-75.00$ \\
Activities & 31.25 & 18.75 & 50.00 & $0-93.75$ \\
Participation & 31.25 & 18.75 & 46.88 & $0-93.75$ \\
\hline
\end{tabular}

Abbreviations: MME, milligrams morphine equivalent; PHQ-9, Patient Health Questionnaire- 9 item; SOAPP-R, Screener and Opioid Assessment for Patients with Pain- Revised; COMM, Current Opioid Misuse Measure; ORT, Opioid Risk Tool; RMDQ-m, modified Roland Morris Disability Questionnaire; WHODAS 2.0, World Health Organization Disability Assessment Schedule 2.0.

There was a statistically significant relationship between responses on the WHODAS 2.0 and RMDQ-m $\left(r_{s}=0.69, p<0.001\right)$. Each of the 6 domains of the WHODAS 2.0 was significantly correlated with the WHODAS 2.0 total, the RMDQ-m, and each of the other WHODAS 2.0 domains at the 0.05 level (Table 3). Both the WHODAS 2.0 and the RMDQ-m were significantly correlated with the PHQ-9 $\left(\mathrm{r}_{\mathrm{s}}=0.68, p<0.001\right.$ and $\mathrm{r}_{\mathrm{s}}=0.60$, $p<0.001$, respectively). Opioid dose was not significantly correlated with the WHODAS $2.0\left(\mathrm{r}_{\mathrm{s}}=0.07, p=0.47\right)$ nor with any other self-report measure.

Subjects' employment status (excluding the Worker's Compensation group because there was only 1 subject in this group) and median disability measure scores are summarized in Table 4. Subjects' self-reported disability was greater for the disabled group when compared with the employed group on both the WHODAS $2.0(p<0.001)$ and the RMDQ-m $(p<0.001)$ and when compared with the retired group on both measures $(p<0.001$ and $p=0.003$, respectively). No other differences in perceived disability among groups reached statistical significance.

There was a statistically significant relationship between subjects' responses on the WHODAS 2.0 and responses on the COMM $\left(\mathrm{r}_{\mathrm{s}}=0.52, p<0.001\right)$ and the SOAPP-R $\left(\mathrm{r}_{\mathrm{s}}=0.51, p<0.001\right)$. There was no significant correlation between patients' responses on the WHODAS 2.0 and the ORT $\left(\mathrm{r}_{\mathrm{s}}=0.14, p=0.12\right)$. Similarly, the correlation between the RMDQ-m and scores on the COMM $\left(\mathrm{r}_{\mathrm{s}}=0.45, p<0.001\right)$ and the SOAPP-R $\left(\mathrm{r}_{\mathrm{s}}=0.42, p<0.001\right)$ were statistically significant, but correlation with ORT did not reach significance $\left(\mathrm{r}_{\mathrm{s}}=0.03, p=0.77\right)$.

\section{Discussion}

The results of this study demonstrated significant positive correlations between self-reported disability as measured by the WHODAS 2.0 and scores on the RMDQ-m. This correlation represents preliminary validation of the WHODAS 2.0 in a novel population of chronic pain patients being considered for COT. All of the 6 subscales were positively correlated to each other and the total WHODAS 2.0 score among our sample. There appears to be good feasibility of the measure for this sample; 109 of 124 patients completed the measure in entirety alongside 4 other screening measures administered. Results did reveal a possible burden as 5 patients left one or more pages incomplete, and another 6 patients left between 3-5 items incomplete, though this study did not gather data on the reasons for incomplete measures.

WHODAS 2.0 scores were not significantly correlated with opioid dose at the time of evaluation, a result consistent with the mixed literature on the impact of opioids on function. ${ }^{4-8}$ Those who had disabled employment status had significantly higher scores on both disability measures compared to employed and retired patients. While level of disability may lead to one applying for disability income, it may be the case that applying for disability income leads to self-perception of worse disability. There may also be other confounding dimensions such as physical impairment or psycho-social difficulties that are measured on the WHODAS 2.0 that affect one's employment status as well. These findings are consistent with a 2010 study in which WHODAS 2.0 scores were significantly different between those working and those not working due to their health condition. ${ }^{31}$

The scores on the WHODAS 2.0 correlated positively with the risk of opioid misuse as measured by the COMM and the SOAPP-R. The ORT screening failed to demonstrate a significant relationship with both disability measures, and may be explained by very recent findings of its questionable validity in a tertiary care chronic pain population. ${ }^{32}$ Consistent with earlier studies identifying a relationship between disability and depression, ${ }^{33-35}$ WHODAS 2.0 scores were positively correlated with PHQ-9 scores.

Given adequate psychometric properties evidenced in the many investigations of the WHODAS 2.0, clinical utility also should be evaluated when one considers the 

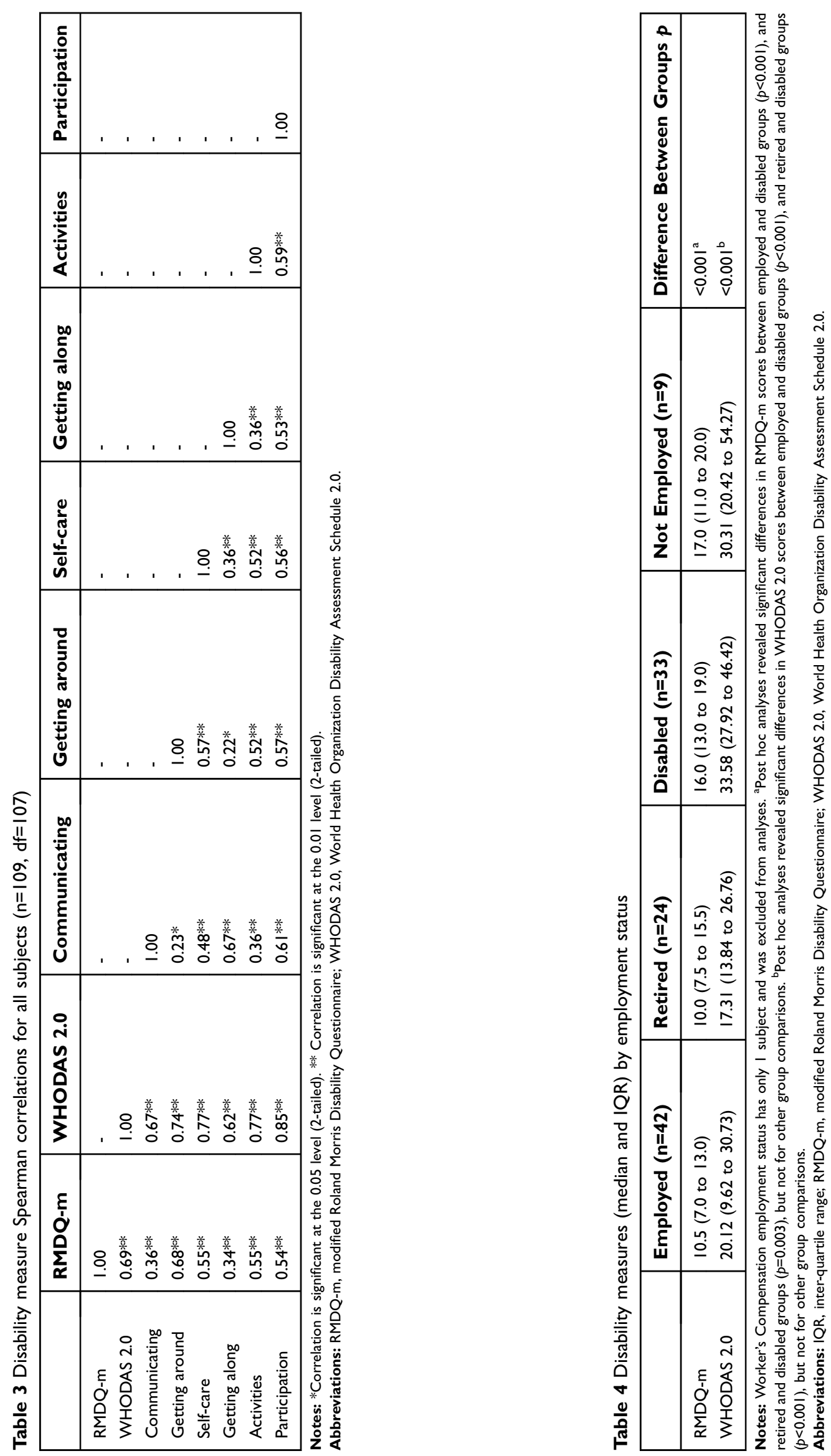
adoption of a self-report disability measure in the context of COT. The WHODAS 2.0 addresses face-valid disability domains that may greatly assist the clinician in goal-setting and measurement of discrete objective gains by the patient. However, other studies have shown that the WHODAS 2.0 suffers from the bias seen with many measures of self-reported disability in that it favors a medical model of disability, over social models or other interpretations, despite the apparent range of items across activities in an attempt to be consistent with the WHO ICF framework of disability. ${ }^{36}$ It is clear that no single instrument can meet all of the required components of a thorough disability assessment, and limitations exist with the WHODAS 2.0 as with any self-report measure. Nonetheless, it remains a promising instrument for clinicians assessing chronic pain in patients receiving COT. In the clinical setting, a simple disability "score" is often inadequate, particularly as the literature and the findings of this study indicate that opioid dose was not correlated with scores on disability measures. Therefore, a disability measure should be considered a clinical tool to use as a component of a thorough disability evaluation to aid in treatment planning.

One of the strengths of this study is that it is the first to our knowledge evaluating the WHODAS 2.0 in a population of chronic pain patients being considered for COT. The study population reflects a broad community-based population seeking pain management at a multidisciplinary, tertiary care pain center for a range of pain complaints, rather than focused on a particular pain-related diagnosis population. Limitations are inherent in retrospective cohort studies, and one common limitation is missing data. This occurred for $12 \%$ of the sample and to address this issue, we used qualitative information about these patients to discuss the feasibility of using the WHODAS 2.0 in this population. Future research should evaluate the feasibility of the 36 -item version compared to the 12-item version, particularly when administered alongside multiple other measures as is typical during opioid risk assessments. Another limitation is that we used the RMDQ-m, another self-report measure of disability, to validate the WHODAS 2.0 in this population, and future validation studies should consider including clinician ratings and/or objective measures of disability and impairment. Given that the use of opioids for chronic pain has been found to be predictive of higher levels of disability as measured by the RMDQ, ${ }^{37}$ our hope is that future research on the WHODAS 2.0 will examine opioids' prediction of higher scores on this measure, as well. Last, this small validation study may not be generalizable to all patients seeking COT at pain management centers, and replication of this study in a larger sample and other settings such as academic hospitals or primary care offices is encouraged.

\section{Conclusion}

The results of this investigation revealed that the WHODAS 2.0 was significantly positively correlated with other measures, including disability, risk of opioid misuse, and depression among patients being evaluated for COT. While not a perfect measure of disability, the WHODAS 2.0 does appear to capture a disability rating based on a broader biopsychosocial definition of disability, as set forth by the WHO ICF. As such, it may be a more useful tool when discussing expectations of both patients and providers at initiation of COT for chronic pain management. This assessment and discussion is crucial, particularly given the focus on function, rather than analgesia alone, when assessing the effectiveness of opioid treatment. 38,39

\section{Acknowledgments}

This research did not receive any specific grant from funding agencies in the public, commercial, or not-forprofit sectors. The abstract of this paper was presented at the 35th Scientific Meeting of the American Pain Society as a poster presentation/conference talk with interim findings. The poster's abstract was published in "Poster Abstracts" in The Journal of Pain: DOI: https://doi.org/ 10.1016/j.jpain.2016.01.097.

\section{Disclosure}

Dr Michael E Schatman is a consultant for Kaleo Pharma, outside the submitted work. The authors report no other conflicts of interest in this work.

\section{References}

1. Institute of Medicine (IOM). Relieving Pain in America: A Blueprint for Transforming Prevention, Care, Education, and Research. Washington, DC: The National Academies Press; 2011.

2. Gaskin DJ, Richard P. The economic costs of pain in the United States. J Pain. 2012;13:715-724. doi:10.1016/j.jpain.2011.12.010

3. Mojtabai R. National trends in long-term use of prescription opioids. Pharmacoepidemiol Drug Saf. 2018;27(5):526-534. doi:10.1002/pds. v27.5

4. Chou R, Turner JA, Devine EB, et al. The effectiveness and risks of long-term opioid therapy for chronic pain: a systematic review for a National Institutes of Health Pathways to Prevention Workshop. Ann Intern Med. 2015;162(4):276-286. doi:10.7326/M14-2559 
5. Dowell D, Haegerich TM, Chou R. CDC guideline for prescribing opioids for chronic pain-United States, 2016. Jama. 2016;315 (15):1624-1645. doi:10.1001/jama.2016.1464

6. Busse JW, Mahmood H, Maqbool B, et al. Characteristics of patients receiving long-term opioid therapy for chronic noncancer pain: a cross-sectional survey of patients attending the Pain Management Centre at Hamilton General Hospital, Hamilton, Ontario. CMAJ Open. 2015;3(3):E324-E330. doi:10.9778/cmajo.20140126

7. Turner JA, Shortreed SM, Saunders KW, LeResche L, Von Korff M. Association of levels of opioid use with pain and activity interference among patients initiating chronic opioid therapy: a longitudinal study. Pain. 2016;157(4):849-857. doi:10.1097/j. pain.0000000000000473

8. Gilliam WP, Craner JR, Cunningham JL, et al. Longitudinal treatment outcomes for an interdisciplinary pain rehabilitation program: comparisons of subjective and objective outcomes on the basis of opioid use status. J Pain. 2018;19(6):678-689. doi:10.1016/j.jpain.2018.02.010

9. Manchikanti L, Kaye AM, Knezevic NN, et al. Responsible, safe, and effective prescription of opioids for chronic non-cancer pain: American Society of Interventional Pain Physicians (ASIPP) Guidelines. Pain Physician. 2017;20(2S):S3-S92.

10. Fairbank JCT, Pynsent PB. The oswestry disability index. Spine. 2000;25:2940-2953. doi:10.1097/00007632-200011150-00017

11. Vernon H, Mior S. The neck disability index: a study of reliability and validity. J Manipulative Physiol Ther. 1991;14:409-415.

12. Budiman-Mak E, Conrad KJ, Roach KE. The foot function index: a measure of foot pain and disability. J Clin Epidemiol. 1991;44:561570. doi:10.1016/0895-4356(91)90220-4

13. McConnell S, Kolopack P, Davis AM. The Western Ontario and McMaster Universities Osteoarthritis Index (WOMAC): a review of its utility and measurement properties. Arthritis Rheum. 2001;45:453-461. doi:10.1002/1529-0131(200110)45:5<453::AIDART365>3.0.CO;2-W

14. Bennett RM, Bushmakin AG, Cappelleri JC, Zlateva G, Sadosky AB. Minimal clinically important difference in the fibromyalgia impact questionnaire. J Rheumatol. 2009;36:1304-1311. doi:10.3899/jrheum.080430

15. Aletaha D, Smolem J. The Simplified Disease Activity Index (SDAI) and the Clinical Disease Activity Index (CDAI): a review of their usefulness and validity in rheumatoid arthritis. Clin Exp Rheumatol. 2005;23(5 Suppl 39):S100-108.

16. Kawata AK, Hsieh R, Bender R, et al. Psychometric evaluation of a novel instrument assessing the impact of migraine on physical functioning: the migraine physical function impact diary. Headache. 2017;57:1385-1398. doi:10.1111/head.13162

17. Brodke DS, Goz V, Voss MW, Lawrence BD, Spiker WR, Hung M. PROMIS PF CAT outperformed the ODI and SF-36 physical function domain in spine patients. Spine. 2017;42(12):921-929. doi:10.1097/ BRS.0000000000001965

18. Chiarotto A, Boers M, Deyo RA, et al. Core outcome measurement instruments for clinical trials in nonspecific low back pain. Pain. 2018;159(3):481-495.

19. Ustün TB, Chatterji S, Kostanjsek N, et al.; WHO/NIH Joint Project. Developing the World Health Organization Disability Assessment Schedule 2.0. Bull World Health Organ. 2010;88:815-823. doi:10.2471/BLT.09.067231

20. Federici S, Bracalenti M, Meloni F, Luciano JV. World Health Organization disability assessment schedule 2.0: an international systematic review. Disabil Rehabil. 2017;39(23):2347-2380. doi:10.1080/09638288.2016.1223177

21. Washington State Agency Medical Directors' Group. Interagency Guideline on Opioid Dosing for Chronic Noncancer Pain: An Educational Aid to Improve Care and Safety with Opioid Treatment. Olympia,WA: Washington State Department of Labor and Industries; 2010.
22. Morris R. A study of the natural history of back pain. Part I: development of a reliable and sensitive measure of disability in low-back pain. Spine. 1983;8(2):141-144. doi:10.1097/00007632-198303000-00004

23. Roland M, Fairbank J. The roland-morris disability questionnaire and the oswestry disability questionnaire. Spine. 2000;25(24):3115-3124. doi:10.1097/00007632-200012150-00006

24. Kroenke K, Spitzer RL, Williams JBW. The PHQ-9: validity of a brief depression severity measure. J Gen Intern Med. 2001;16:606613. doi:10.1046/j.1525-1497.2001.016009606.x

25. Butler SF, Budman SH, Fernandez K, Jamison RN. Validation of a screener and opioid assessment measure for patients with chronic pain. Pain. 2004;112:65-75. doi:10.1016/j.pain.2004.07.026

26. Butler SF, Fernandez K, Benoit C, Budman SH, Jamison RN. Validation of the revised Screener and Opioid Assessment for Patients with Pain (SOAPP-R). J Pain. 2008;9:360-372. doi:10.1016/j.jpain.2007.11.014

27. Butler SF, Budman SH, Fernandez KC, Fanciullo GJ, Jamison RN. Cross-validation of a screener to predict opioid misuse in chronic pain patients (SOAPP-R). J Addict Med. 2009;3:66-73. doi:10.1097/ ADM.0b013e318185e71d

28. Butler SF, Budman SH, Fernandez KC, et al. Development and validation of the current opioid misuse measure. Pain. 2007;130:144-156. doi:10.1016/j.pain.2007.01.014

29. Butler SF, Budman SH, Fanciullo GJ, Jamison RN. Cross validation of the current opioid misuse measure to monitor chronic pain patients on opioid therapy. Clin J Pain. 2010;26:770-776. doi:10.1097/ AJP.0b013e3181f195ba

30. Webster LR, Webster RM. Predicting aberrant behaviors in opioidtreated patients: preliminary validation of the Opioid Risk Tool. Pain Med. 2005;6:432-442. doi:10.1111/j.1526-4637.2005.00072.x

31. Garin O, Ayuso-Mateos JL, Almansa J, et al. Validation of the "World Health Organization Disability Assessment Schedule, WHODAS-2" in patients with chronic diseases. Health Qual Life Outcomes. 2010;8(51):1-15. doi:10.1186/1477-7525-8-51

32. Clark MR, Hurley RW, Adams MCB. Re-assessing the validity of the Opioid Risk Tool in a tertiary academic pain management center population. Pain Med. 2018;19:1382-1395. [Epub ahead of print]. doi:10.1093/pm/pnx332

33. Schneider M, Baron E, Davies T, Bass J, Lund C. Making assessment locally relevant: measuring functioning for maternal depression in Khayelitsha, Cape Town. Soc Psychiatry Psychiatr Epidemiol. 2015;50:797-806. doi:10.1007/s00127-014-1003-0

34. Srifuengfung M, Noppakun K, Srisurapanont M. Depression in kidney transplant recipients: prevalence, risk factors, and association with functional disabilities. J Nerv Ment Dis. 2017;205:788-792. doi:10.1097/NMD.0000000000000722

35. Antunes A, Frasquilho D, Azeredo-Lopes S, et al. Disability and common mental disorders: results from the world mental health survey initiative portugal. Eur Psychiatry. 2018;49:56-61. doi:10.1016/j.eurpsy.2017.12.004

36. Kulnik ST, Nikoletou D. WHODAS 2.0 in community rehabilitation: a qualitative investigation into the validity of a generic patientreported measure of disability. Disabil Rehabil. 2013;36(2):146154. doi:10.3109/09638288.2013.851742

37. Ashworth J, Green DJ, Dunn KM, et al. Opioid use among low back pain patients in primary care: is opioid prescription associated with disability at 6-month follow-up? Pain. 2013;154:1038-1044. doi:10.1016/j.pain.2013.03.011

38. Ballantyne JC, Sullivan MD. Intensity of chronic pain-the wrong metric? N Engl J Med. 2015;373:2098-2099. doi:10.1056/NEJMp1507136

39. Sullivan MD, Ballantyne JC. Must we reduce pain intensity to treat chronic pain? Pain. 2016;157:65-69. doi:10.1097/j.pain.000 0000000000473 


\section{Publish your work in this journal}

The Journal of Pain Research is an international, peer reviewed, open access, online journal that welcomes laboratory and clinical findings in the fields of pain research and the prevention and management of pain. Original research, reviews, symposium reports, hypothesis formation and commentaries are all considered for publication. The manuscript management system is completely online and includes a very quick and fair peer-review system, which is all easy to use. Visit http:// www.dovepress.com/testimonials.php to read real quotes from published authors.

Submit your manuscript here: https://www.dovepress.com/journal-of-pain-research-journal 